\title{
Amphotericin B-Related Nephrotoxicity in Low-Risk Patients
}

\author{
Roberto Herz Berdichevski. ${ }^{1,3}$, \\ Luciano Billodre Luis ${ }^{2}$, Letícia Crestana ${ }^{2}$ \\ and Roberto Ceratti Manfro ${ }^{1,2,3}$
}

\author{
${ }^{1}$ Post Graduate Medical Sciences: Nephrology Program; \\ ${ }^{2}$ Department of Internal Medicine, School of Medicine, Federal \\ University of Rio Grande do Sul; ${ }^{3}$ Division of Nephrology, \\ Hospital de Clínicas de Porto Alegre; Porto Alegre, RS, Brazil
}

\begin{abstract}
Introduction. Amphotericin B (AmphoB) is the drug of choice for treatment of severe fungal illnesses; however, it is very nephrotoxic. Modified (less toxic) amphotericins are very expensive. In low-risk patients, saline loading would be enough to prevent significant loss of renal function. Material and Methods. Patients with normal renal function and within the first 24 hours of treatment with AmphoB were prospectively enrolled in the study. Patients in intensive care units or who were using vasoactive drugs were excluded. Saline loads were infused before and after the AmphoB treatment. Blood and urine analyses were made at the beginning and at the end of the treatment. Serum creatinine was repeated 30 days after the end of the AmphoB treatment. Results. The mean increase in serum creatinine in the 48 patients was $0.3(0.18-0.41) \mathrm{mg} / \mathrm{dL}$, due to a mean decrease of $25(12.8$ 36.9) $\mathrm{mL} / \mathrm{min}$ of creatinine clearance $(\mathrm{CrCl})$. Acute renal failure, defined as an increase of more than $50 \%$ of the baseline creatinine, occurred in 15 patients $(31 \%)$. Patients that were on antibiotics, in post-chemotherapy status or those submitted to bone marrow transplantation had the highest risk. Mean serum creatinine and the $\mathrm{CrCl}$ levels were no different from baseline values after 30 days. Conclusion. In low-risk patients, the use of AmphoB with prophylactic sodium chloride loading was associated with a small and reversible decrease in renal function. Due to its high cost the use of more expensive therapies for this type of patient does not seem to be justified.

Key Words: Amphotericin B, acute renal failure, nephrotoxicity, sodium chloride.
\end{abstract}

Despite the high incidence of adverse effects associated with its use, amphotericin $\mathrm{B}(\mathrm{AmphoB})$ remains the drug of choice for the treatment of severe fungal infections [1]. AmphoB-induced nephrotoxicity occurs in around $80 \%$ of treated patients; it is associated with a high morbidity rate $[1,2]$. The two main renal manifestations of AmphoB-related nephrotoxicity are reduction of the glomerular filtration rate and tubular dysfunction. The glomerular filtration rate decreases due to renal vasoconstriction during drug infusion, and tubular dysfunction is a consequence of direct lesion of the cellular membrane due to interaction of the AmphoB molecule with cholesterol from the tubular cell membranes [36]. The interaction between these two mechanisms occurs through a tubuloglomerular feedback, in which low sodium delivery at the macula densa, caused by proximal tubular dysfunction, enhances afferent vasoconstriction, reducing renal blood flow. This mechanism is enhanced in hypovolemic individuals, and vasoconstriction itself may in turn increase tubular injury [7].

Received on 27 September 2005; revised 16 March 2006.

Address for correspondence: Dr. Roberto H. Berdichevski. Rua Cabral 1390/201, 90440090 Porto Alegre, RS, Brazil. Telephone: +55 51- 30245544 / 51- 99943914. Fax : +55 5121018295 .

E-mail: roberto.herz@terra.com.br. Financial Support: This paper received financial support from the Brazilian National Council of Technological and Scientific Development (CNPq).

The Brazilian Journal of Infectious Diseases 2006;10(2):94-99. (C) 2006 by The Brazilian Journal of Infectious Diseases and Contexto Publishing. All rights reserved.
The growing number of high-risk patients, such as those with acquired immunodeficiency syndrome (AIDS), patients needing immunosuppressive therapy or chemotherapy, and patients treated in intensive care units, has created a particularly susceptible population for fungal infections, making the use of AmphoB more common, and thus increasing the number of patients at risk for renal dysfunction. Several studies published during the last two decades have shown that nephrotoxicity can be prevented by the use of sodium loading [4,7-11], slowing drug infusion [12] and through the use of liposomal or lipid-complex amphotericins [13-17].

Based on the results of clinical trials, liposomal amphotericin has been recommended for the prevention of acute renal failure (ARF) [13-17]. These studies included a largely unselected population of patients, with many of them being treated at intensive care units, using vasoactive drugs, and with multiple risk factors for developing ARF. However the cost of treatment with modified amphotericins is significantly higher than with amphotericin deoxycholate. Moreover, it has not been evaluated whether their use in lowrisk patients would be of significant benefit. We examined the incidence and severity of AmphoB-induced renal dysfunction in hemodynamically stable patients with initial normal renal function who were submitted to saline loading to reduce AmphoB nephrotoxicity.

\section{Material and Methods}

We prospectively evaluated consecutive in-hospital patients who initiated AmphoB treatment at the Hospital de 
Clínicas de Porto Alegre from March 2002 through August 2003. The inclusion criteria were: age over 12 years, normal renal function, and being within the first 24 hours of treatment with AmphoB. Potential patients for the study were identified by the research team with the help of the hospital pharmacy registry, so that every patient initiating AmphoB would be identified. The exclusion criteria were: patients in intensive care units, with hemodynamic instability (defined by the need for vasoactive drugs) and with serum creatinine levels higher than $1.3 \mathrm{mg} / \mathrm{dL}$.

All patients were informed of the objectives of the study and signed an informed consent. This study was approved by the Research Ethics Committee of the Hospital de Clínicas de Porto Alegre, accredited by the Research Ethics National Committee (CONEP), Brazilian Health Ministry, and registered with the Office for Human Research Protections (OHRP-USDHHS) as an Institutional Review Board (IRB 00000921).

All patients received a one-liter infusion of $0.9 \%$ sodium chloride, divided into $500 \mathrm{~mL}$ before and $500 \mathrm{~mL}$ after AmphoB infusion. This drug was diluted in 5\% glucose at a concentration of $0.1 \mathrm{mg} / \mathrm{mL}$ and infused over a four-hour period. The dose $(0.5-1.0 \mathrm{mg} / \mathrm{kg} / \mathrm{day})$, the duration of the treatment, eventual withholding of AmphoB treatment, as well as handling of electrolytic disturbances, were decided and implemented by the attending medical teams and were not influenced by the study protocol. Collected data consisted of gender, age, race (defined by the patients at the moment of the interview), weight, diagnosis, indication for use of AmphoB, presence and localization of the fungal infection, antibiotics used during the previous week, clinical co-morbidities, use of any other potentially nephrotoxic drug, blood pressure, and urinary output. Blood and urine samples were collected at the beginning of and after 14 days of AmphoB treatment, or at its end or discontinuation. Serum concentrations of creatinine, urea, potassium, bicarbonate and sodium were determined by automated methods. $\mathrm{pH}$ and sediment analysis of urine were performed, as well as measurements of urinary sodium, potassium, proteins and creatinine. Creatinine clearance $(\mathrm{CrCl})$ was estimated by using the Cockcroft-Gault method; the excretion fractions of sodium (FENa) and potassium (FEK), as well as the proteincreatinine index (PCI), were measured. Another serum creatinine measurement was made 30 days after the end of the treatment.

Data are presented as mean \pm standard deviation, or as percentages. Paired t tests were used for the comparisons of continuous variables. Ninety-five percent confidence intervals were calculated. Pearson's correlation coefficients were also calculated. Chi-square, Fisher's tests and simple or multiple Poisson regressions were used for the categorical variables. A P level lower than 0.05 was required for statistical significance. The softwares SPSS for Windows version 8.0, PEPI and STATA 7.0 were used for the analyses.

\section{Results}

Forty-eight patients were included in the study. Thirtytwo $(67 \%)$ were males and $39(81 \%)$ were white. The mean age was $39.6 \pm 13.9$ years old (Table 1). Most of the patients were attended by the hematology $(48 \%)$ and internal medicine/ infectious disease $(44 \%)$ teams. Patients were treated for an average period of $8.6 \pm 3.3$ days, having received a cumulative dose of $411.5 \pm 197 \mathrm{mg}$ or $6.25 \pm 2.9 \mathrm{mg} / \mathrm{kg}$. The site of infection was not identified in 16 cases $(33 \%)$, predominantly in the group of patients with febrile neutropenia (12/21). Central nervous system infections occurred in 15 cases (31\%), mainly in patients with AIDS (15/20). Mean arterial pressure (MAP) was $86 \pm 12 \mathrm{mmHg}$. Thirty-seven patients (77\%) were receiving antibiotics when AmphoB was started. A recognized nephrotoxic agent was being used in 19 (40\%), vancomycin being the most frequent ( 15 cases; $31 \%$ ).

Table 1. Demographics and general characteristics of the patients

\begin{tabular}{|c|c|}
\hline Number of patients & 48 \\
\hline Age (years) & $39.7 \pm 13.8$ \\
\hline Male & $32(67 \%)$ \\
\hline \multicolumn{2}{|l|}{ Race } \\
\hline White & $39(81 \%)$ \\
\hline Non-white & $9(19 \%)$ \\
\hline \multicolumn{2}{|l|}{ Initial diagnosis } \\
\hline Post-Chemotherapy & $21(44 \%)$ \\
\hline AIDS & $20(42 \%)$ \\
\hline BMT & $4(8.3 \%)$ \\
\hline Systemic candidiasis & $2(4.2 \%)$ \\
\hline Fungal identification at treatment beginning & $16(33 \%)$ \\
\hline \multicolumn{2}{|l|}{ Site of fungal infection (proved or suspected) } \\
\hline Non-identified focus & $16(33 \%)$ \\
\hline Central Nervous System & $15(31 \%)$ \\
\hline Lungs & $09(19 \%)$ \\
\hline Skin & $02(4.2 \%)$ \\
\hline Pharmacological immunosuppression* & $25(52 \%)$ \\
\hline \multicolumn{2}{|l|}{ Co-morbidities } \\
\hline Hypertension $(>140 / 90 \mathrm{mmHg})$ & $06(13 \%)$ \\
\hline Previous nephropathy & $02(4.2 \%)^{* *}$ \\
\hline Diabetes mellitus & $01(2.1 \%)$ \\
\hline Antibiotics use (current or on the last week) & $37(77 \%)$ \\
\hline \multicolumn{2}{|l|}{ Nephrotoxic drugs use } \\
\hline Vancomicin & $15(31 \%)$ \\
\hline IV Acyclovir & $03(6.3 \%)$ \\
\hline Captopril & $01(2.1 \%)$ \\
\hline Mean Arterial Pressure (mmHg) & $86 \pm 11.9$ \\
\hline
\end{tabular}

Data are presented as mean \pm standard-deviation and as percentages. AIDS - Acquired Immunodeficiency Syndrome.

BMT - Bone Marrow Transplantation. (*) Status after chemotherapy, cyclosporin or steroid use. $(* *)$ Urinary stone disease, pyelonephritis. Serum creatinine level $<1.3 \mathrm{mg} / \mathrm{dL}$. 
Table 2. Comparison of renal function before and after amphotericin B treatment

\begin{tabular}{lcccc}
\hline & Before treatment & After treatment & $\begin{array}{c}\text { Differences between means } \\
(\mathbf{C I ~ 9 5 \% ) ~}\end{array}$ & $\begin{array}{c}\text { P } \\
\text { value }\end{array}$ \\
\hline Serum creatinine $(\mathrm{mg} / \mathrm{dL})$ & $0.8 \pm 0.2$ & $1.1 \pm 0.4$ & $0.29(0.18-0.41)$ & $<0.001$ \\
$\mathrm{CrCl}(\mathrm{mL} / \mathrm{min})^{(*)}$ & $112.1 \pm 38.6$ & $87.3 \pm 32.8$ & $24.9(12.8-36.9)$ & $<0.001$ \\
Plasmatic Urea $(\mathrm{mg} / \mathrm{dL})$ & $34.4 \pm 15.2$ & $49.5 \pm 33.4$ & $15.1(7.1-23.1)$ & $<0.001$ \\
Serum Potassium $(\mathrm{mEq} / \mathrm{L})$ & $3.8 \pm 0.7$ & $3.6 \pm 0.9$ & $0.13(-0.4-0.9)$ & -0.33 \\
Serum Magnesium $(\mathrm{mEq} / \mathrm{L})$ & $1.8 \pm 0.3$ & $1.8 \pm 0.4$ & $0.004(0.13-0.14)$ & 0.94 \\
Serum Bicarbonate $(\mathrm{mEq} / \mathrm{L})$ & $28.3 \pm 3.8$ & $25.9 \pm 7.1$ & $2.4(0.4-4.4)$ & 0.01 \\
Urinary pH & $6.6 \pm 0.8$ & $6.7 \pm 0.8$ & $0.05(-0.27-0.38)$ & 0.73 \\
Urinary potassium $(\mathrm{mEq} / \mathrm{L})$ & $36.1 \pm 21.3$ & $33.0 \pm 15.5$ & $3.1(-4.7-10.9)$ & 0.42 \\
Urinary sodium $(\mathrm{mEq} / \mathrm{L})$ & $81.0 \pm 29.2$ & $88.6 \pm 30.8$ & $7.6(-24.10-8.84)$ & 0.35 \\
FE sodium $(\%)^{(*)}$ & $1.5 \pm 1.17$ & $3.4 \pm 2.73$ & $1.98(1.02-4.19)$ & $<0.001$ \\
FE potassium $(\%)^{(* *)}$ & $18.7 \pm 10.2$ & $48.2 \pm 37.5$ & $29.6(17.7-41.5)$ & $<0.001$ \\
Protein/creatinine ratio & $0.9 \pm 1.2$ & $2.0 \pm 1.7$ & $1.1(0.56-4.08)$ & $<0.001$ \\
\hline Part & & &
\end{tabular}

Paired t-test was used for comparison of the continuous variables. Data are shown as mean (standard-deviation), mean differences and confidante interval of 95\%. (*) Estimated Creatinine Clearance-Cockcroft-Gault formula. (**) Fractional Excretion Rate.

Table 3. Univariate analysis of risk factors for acute renal failure

\begin{tabular}{|c|c|c|c|c|c|}
\hline & Mean \pm SD & Mean of the differences & $\mathbf{r}$ & $95 \% \mathrm{CI}$ & Pvalue \\
\hline \multicolumn{6}{|l|}{ Gender } \\
\hline $\mathrm{M}$ & $0.32 \pm 0$ & 0.07 & - & $-0.18 ; 0.32$ & $0.5^{(*)}$ \\
\hline $\mathrm{F}$ & $0.25 \pm 0.4$ & & & & \\
\hline \multicolumn{6}{|l|}{ Race } \\
\hline White & $0.3 \pm 0.4$ & 0.05 & - & $-0.36 ; 0.25$ & $0.7^{(*)}$ \\
\hline Non-white & $0.3 \pm 0.4$ & & & & \\
\hline \multicolumn{6}{|l|}{ Antibiotic use } \\
\hline Yes & $0.3 \pm 0.4$ & 0.26 & - & $-0.05 ; 0.59$ & $0.11^{(*)}$ \\
\hline No & $0.05 \pm 0.2$ & & & & \\
\hline \multicolumn{6}{|l|}{ Nephrotoxic drug use } \\
\hline Yes & $0.33 \pm 0.4$ & 0.04 & - & $-0.2 ; 0.29$ & $0.69^{(*)}$ \\
\hline No & $0.28 \pm 0.4$ & & & & \\
\hline Cumulative dose & - & - & 0.006 & $-0.91^{(* *)}$ & \\
\hline Mean Arterial Pressure & - & - & 0.017 & - & $0.97^{(\varnothing)}$ \\
\hline Age & - & - & 0.57 & $-0.71^{(\varnothing)}$ & \\
\hline \multicolumn{6}{|l|}{ Initial Diagnosis } \\
\hline AIDS & $0.2 \pm 0.4$ & - & - & $-0.21^{(\varnothing \varnothing)}$ & \\
\hline Post-ChThr & $0.4 \pm 0.4$ & & & & \\
\hline Others & $0.4 \pm 0.4$ & & & & \\
\hline
\end{tabular}

(*) t- test for independent samples; (**) Spearman's correlation; (ØØ) Pearson's correlation; (ØØ) Analysis of variance (ANOVA). ChThr- Chemotherapy; CI - confidence interval

Twenty-five patients (52\%) completed their treatments within the time initially established by the medical teams. The reasons for premature ending of treatment in the others were: adverse effects attributed to AmphoB (7 cases; 14.5\%), identification of a bacteria ( 6 cases; $12.5 \%$ ) and patient death ( 5 cases; $10.5 \%$ ). Renal function at the beginning and at the end of the treatment is shown in Table 2. Serum creatinine and urea levels increased significantly over time. Estimated creatinine clearance and serum bicarbonate had significant mean reductions of $25 \mathrm{~mL} / \mathrm{min}$ and $2.4 \mathrm{mEq} / \mathrm{L}$ respectively. No alterations were observed in serum potassium, magnesium or in urinary $\mathrm{pH}$. There was tubular dysfunction, as demonstrated by the alterations in FENa and FEK. Patients received an average of $74 \mathrm{mEq} /$ day or $644 \mathrm{mEq}$ - total of oral or intravenous potassium.

Table 3 shows the univariate analysis of the variables potentially associated with serum creatinine level changes. No association was observed between age, sex, race, use of nephrotoxic drugs, use of antibiotics, or cumulative dose, and mean arterial pressure or serum creatinine changes. Patients with concomitant use of antimicrobials presented a trend towards increasing serum creatinine, when compared with 
Table 4. Analysis of variables associated with acute renal failure (ARF) after amphotericin B treatment

\begin{tabular}{|c|c|c|c|c|c|}
\hline & \multirow[t]{2}{*}{$\operatorname{ARF}(\%)$} & \multicolumn{2}{|l|}{ Univariate } & \multicolumn{2}{|c|}{ Multivariate } \\
\hline & & $\mathbf{R R}^{1}(\mathbf{C I} 95 \%)$ & Pvalue & $\mathbf{R R}^{2}(\mathbf{C I} 95 \%)$ & Pvalue \\
\hline \multicolumn{6}{|l|}{ Gender* } \\
\hline $\mathrm{F}$ & 31.3 & $1.3(0.4-4.3)$ & 1.00 & - & - \\
\hline M & 31.3 & & & & \\
\hline \multicolumn{6}{|l|}{ Race $^{* *}$} \\
\hline White & 33.3 & $1.7(0.32-9.7)$ & 0.7 & - & - \\
\hline Non-white & 22.2 & & & & \\
\hline \multicolumn{6}{|l|}{ Antibiotics use ${ }^{* *}$} \\
\hline Yes & 40.5 & Infinite $¥$ & 0.01 & - 佯 & - \\
\hline No & 0 & & & & \\
\hline \multicolumn{6}{|c|}{ Nephrotoxic drugs use ${ }^{* *}$} \\
\hline Yes & 47.4 & $2.3(0.97-5.4)$ & 0.06 & $1.5(0.6-3.6)$ & 0.42 \\
\hline No & 20.7 & & & & \\
\hline \multicolumn{6}{|l|}{ Initial diagnosis } \\
\hline AIDS & 10 & $1.0^{* * * *}$ & & $1.0^{* * * *}$ & \\
\hline After-ChTh & 42.9 & $4.3(1.1-17.7)$ & 0.02 & $3.6(0.8-17.1)$ & 0.1 \\
\hline Others & 57.1 & $5.7(1,3-25.1)$ & & $4.8(0.96-24.5)$ & 0.057 \\
\hline $\operatorname{Age}^{* * *}$ & - & $1.0(0.96-1.03)$ & 0.6 & - & - \\
\hline MAP $^{* * *}$ & - & $1.0(0.95-1.03)$ & 0.7 & - & - \\
\hline Cumulative dose $\mathrm{e}^{* * * *}$ & - & $1.0(0.9-1.05)$ & 0.2 & - & - \\
\hline
\end{tabular}

patients who were not using antibiotics. Although patients were significantly more proteinuric after treatment, no association was observed between the protein-creatine ratio and serum creatinine changes (data not shown).

The incidence of ARF, as defined by an increase of serum creatinine of at least $50 \%$ of the baseline value, was observed in 15 cases $(31 \%)$. The uni- and multivariate analyses of the factors associated with the development of ARF are shown in Table 4. Based on univariate analysis, the use of antibiotics, the initial diagnosis and the use of nephrotoxic drugs, were all associated with the development of ARF, which did not develop in any patient not receiving antibiotics. Patients in the post-chemotherapy period and with other relevant conditions (bone marrow transplants, systemic candidemia) also presented elevated incidences of ARF. Age, MAP, cumulative dose, sex and race were not significantly correlated with the development of ARF. Based on the multivariate analysis, only the initial diagnosis remained associated with the development of ARF. One patient $(2.1 \%)$ had a greater than $2.0 \mathrm{mg} / \mathrm{dL}$ increase of creatinine . Another patient developed ARF in association with sepsis and multiple organ dysfunctions and needed dialysis support 21 days after the discontinuation of AmphoB therapy. At the time of ending AmphoB treatment, his serum creatinine was $1.5 \mathrm{mg} / \mathrm{dL}$.

Thirty-seven patients were followed-up for 30 days after the end of AmphoB treatment. Mean serum creatinine levels before and after treatment in this group of patients were $0.8 \pm$ 0.2 and $0.9 \pm 0.3 \mathrm{mg} / \mathrm{dL}$, respectively, (difference: $0.1 ; 95 \%$ confidence interval (CI): $-0.21-0.01 \mathrm{mg} / \mathrm{dL} ; \mathrm{p}=0.7)$. In these patients the mean $\mathrm{CrCl}$ value 30 days after the end of $\mathrm{AmphoB}$ therapy was $106 \pm 35 \mathrm{~mL} / \mathrm{min}$, which is not significantly different from the baseline mean value of $116 \pm 40 \mathrm{~mL} / \mathrm{min}$ (difference: 10.5; 95\%CI: $-5.3-26.7 \mathrm{~mL} / \mathrm{min} ; \mathrm{p}=0.18$ ).

\section{Discussion}

AmphoB-related nephrotoxicity is an important cause of morbidity, occurring to some degree in up to $80 \%$ of patients treated with this drug $[1,2]$. Among the many experimentallytested prophylactic methods, only saline overloading and the use of modified forms of amphotericin (liposomal, lipidcomplex, cholesteryl -sulphate) have been successful in clinical trials. Infusion during 24 hours of AmphoB has also proven effective for the reduction of nephrotoxicity [12].

The use of modified amphotericins has increased progressively over the last years. This practice is based upon the results of clinical trials that have demonstrated reductions of $50-75 \%$ in the incidence of ARF [13-17]. In these studies the development of ARF, usually defined as a $50 \%$ rise in the basal serum creatinine levels and a peak creatinine level higher than $2.0 \mathrm{mg} / \mathrm{dL}$, occurred in $34 \%$ to $49 \%$ of the patients. The lowest reported incidence of ARF in patients who used 
modified amphotericins was 9\% [16]. However, the high economic cost of treatment with modified amphotericins is an important obstacle to their wider use. In Brazil, the daily cost of liposomal amphotericin ranges from US\$179 to 358, much more expensive than AmphoB deoxycholate, which costs US\$ 4.20 per day.

Clinical and experimental data from the last decade point to an important effect of saline loading on the prevention of the loss of renal function in individuals treated with AmphoB. In two trials, there was no significant loss of renal function in sodium chloride-loaded patients $[8,10]$.

The reasons for the discrepancy in the success of such treatments could be due to differences in the study populations. During the last decades, patient profiles and the complexity of their conditions have increased significantly. The spectrum of AmphoB use in patients admitted to the hospital is very heterogeneous. Studies with modified amphotericins have used unselected patients, including those admitted to intensive care treatment centers, who are hemodynamically unstable, with high risk for the development of ARF. In such a scenario, AmphoB could act as a co-factor. Given the high degree of morbidity and the increase in mortality resulting from critical care patients who develop ARF, it seems appropriate, based on the results of clinical trials, to use modified amphotericins in this group of patients. However the need for their use in patients with low risk for ARF has not been clearly defined.

In this study, we sought to better understand renal function in patients with previously-normal renal function, who had no hemodynamic instability and who were not interned in critical care units. A sample of relatively young patients, with few degenerative diseases (diabetes, hypertension, or ischemic heart disease) resulted from this selection. Nonetheless, this population had serious clinical conditions, with a mortality rate of $10 \%$ within this short period. During treatment, significant reductions in the estimated $\mathrm{CrCl}$ and increases in the baseline creatinine were observed. We recognize that the physiological impact of these changes is significant; however, at least in the short term, there were no severe clinical implications. The incidence of ARF, as defined here, was $31 \%$. Our analysis of the conditions associated with the development of ARF uncovered the use of antibiotics as being the most relevant. Actually, there were no cases of ARF in patients who were not receiving antibiotics. The patient's main diagnosis was also related to the development of ARF, and the use of nephrotoxic drugs had a borderline relationship with progression to ARF. It is possible that the use of antibiotics is the determinant factor for the development of ARF in patients with clinical conditions prone to this complication. Patients in post-chemotherapy status or who have been submitted to bone marrow transplants accounted for $73 \%$ of the patients using antibiotics, and these were the groups with positive associations with ARF occurrence. On the other hand, $90 \%$ of the patients with AIDS were not using antibiotics, and they were the group with the lowest incidence of ARF. A relationship between the use of antibiotics and nephrotoxic drugs also exists. Among the usually-considered nephrotoxic drugs, we observed that vancomycin was the most commonly used. Vancomycin was also used in half of the patients who were on antibiotics, mainly because it is used as a second line agent for febrile neutropenia (for febrile neutropenic patients non-responsive to cefepime). We did not include antibiotic use as a variable in the multivariate model, due to the fact that there was no ARF in the group that did not receive antibiotics.

Usually the literature on ARF in patients using AmphoB defines ARF as an increase of the serum creatinine above 2.0 $\mathrm{mg} / \mathrm{dL}$. Using this criterion, the incidence in our study was only $2.1 \%$. Only one patient needed dialysis, and that occurred after amphotericin treatment had been discontinued, within a context of multiple organ dysfunction.

In our study group, the estimated creatinine clearance returned to the initial values within 30 days after the end of AmphoB treatment. This finding strongly suggests that renal dysfunction in these patients was small and reversible. The cumulative dose of amphotericin could, perhaps, explain the low incidence of renal alterations. However, in a large retrospective study including more than 600 patients [18], the sub-group of patients that received less than 500 $\mathrm{mg} /$ period of AmphoB had an ARF incidence of $22 \%$, defined by doubling of the serum creatinine, with a peak of at least $3.0 \mathrm{mg} / \mathrm{dL}$. In another study with non-selected patients that compared AmphoB with liposomal amphotericin, the incidence of ARF, defined by an increase of $100 \%$ in the final creatinine levels, when compared to the initial levels, was $50 \%$ in the group that received AmphoB, with a cumulative dose of $6.18 \mathrm{mg} / \mathrm{kg}$, a dosage very similar to the one received by the patients in our study [16]. Using this criterion in our sample, only $16.7 \%$ would have ARF. The tubular alterations caused by the use of AmphoB still do not have an effective prophylaxis. In our study, tubular dysfunction was evidenced by alterations in FENa and FEK, with a slight trend towards acidosis, but it did not result in relevant hydro-electrolytic disturbances, probably because of the skilled handling of potassium and magnesium during treatment. Some patients were given preventive doses of potassium. This fact also must be taken in consideration when one evaluates glomerular filtration, since there is convincing evidence that hypokalemia can potentiate other injuries to the kidney $[19,20]$.

Finally, we concluded that in patients with a low-risk profile, defined as normal renal function, hemodynamic stability and not being in intensive care therapy, the use of AmphoB with prophylactic administration of $0.9 \%$ sodium chloride was associated with only a small degree of reversible damage to renal function. Due to their high cost in our country, the use of more expensive agents in this group of patients does not seem to be justified at the moment; this concept should be further evaluated with randomized studies. 


\section{References}

1. Mandell G.L., Petri W.A. Jr. Antimicrobial Agents. In: Hardman J.G., Limbird L.E. eds. Goodman and Gilman's - The Pharmacological Basis of Therapeutics. New York, US: McGraw-Hill, 1996;1165-8.

2. Patel R. Antifungal agents - Part I- Amphotericin B preparations and flucytosine. Mayo Clin Proc 1998; 73:1205-25.

3. Sawaya B.P., Briggs J.P., Schnermann J. Amphotericin B nephrotoxicity: the adverse consequences of altered membrane properties. J Am Soc Nephrol 1995;6:154-64.

4. Tolins J.P., Raij L. Chronic amphotericin B nephrotoxicity in the rat, protective effect of prophylactic salt loading. Am J Kidney Dis 1988;11:313-7.

5. Sabra R., Takahashi K., Branch R.A., Badr K.F. Mechanisms of amphotericin B-induced reduction of the glomerular filtration rate: a micropuncture study. J Pharmacol Exp Therap 1990; $253: 34-7 ; 65: 500-6$.

6. Bhathena D.B., Bullock W.E., Nuttall C.E., Luke R.G. The effects of amphotericin B therapy on the intrarenal vasculature and renal tubules in man. Clin Nephrol 1978;9:103-10.

7. Hedemann H.T., Gerkens J.F., Spickard W.A. Amphotericin B nephrotoxicity in humans decreased by salt repletion. Am J Med 1983;75:476-81.

8. Arning M., Scharf R.E. Prevention of amphotericin B-induced nephrotoxicity by loading with sodium chloride: a report of 1291 days of treatment with amphotericin B without renal failure. Klin Wochenchr 1989;67:1020-8.

9. Llanos A., Cieza J., Bernardo J., Echevarria J., et al. Effect of salt supplementation on amphotericin B nephrotoxicity. Kidney Int 1991;40:302-8.

10. Mayer J., Doubek M., Doubek J., et al. Reduced nephrotoxicity of conventional amphotericin B therapy after minimal nephroprotective measures: animal experiments and clinical study. J Infect Dis 2002 Aug 1;186:379-88.

11. Branch R.A., Jackson E.K., Jacqz E., et al. Amphotericin-B nephrotoxicity in humans decreased by sodium supplements with coadministration of ticarcillin or intravenous saline. Klin Wochenschr 1987;65:500-6.
12. Ericksson U., Burkhard S., Schaffner A. Comparison of effects of amphotericin B deoxicolate infused over 4 or 24 hours: randomized controlled trial. BMJ 2001;322:16.

13. Nucci M., Loureiro M., Silveira F., et al. Comparison of the toxicity of amphotericin $\mathrm{B}$ in $5 \%$ dextrose with that of amphotericin in fat emulsion in a randomized trial with cancer patients. Antimicrob Agents Chemother 1999; 43:1445-8.

14. Johnson P.C., Wheat L.J., Cloud G.A., et al. Safety and efficacy of liposomal amphotericin B compared with conventional amphotericin for induction therapy of histoplasmosis in patients with AIDS. Ann Intern Med 2002; 137:105-9.

15. Prentice H.G., Hann I.M., Herbrecht R., et al. A randomized comparison of liposomal versus conventional amphotericin B for the treatment of pyrexia of unknown origin in neutropenic patients. Br J Haemathol 1997;98:711-8.

16. Walsh T.J., Finberg R.W., Arndt C., et al. Liposomal amphotericin B for empirical therapy in patients with persistent fever and neutropenia. National Institute of Allergy and Infectious Diseases Mycoses Study Group. New Engl J Med 1999;340:764-71.

17. Wingard J.R., White M.H., Anaissie E.,et al. A randomized, double-blind comparative trial evaluating the safety of lipossomal amphotericin B versus amphotericin B lipid complex in the empirical treatment of febrile neutropenia. $\mathrm{L}$ Amph/ABLC Collaborative Study Group. Clin Infec Dis 2000;31:1155-63.

18. Bates D.W., Su L., Yu D.T., et al. Correlates of acute renal failure in patients receiving parenteral amphotericin B. Kidney Int 2001;60:1452-9.

19. Seguro A.C., Shimizu M.H., Monteiro J.L., et al. Effect of potassium depletion in ischemic renal failure. Nephron 1989;51:350-4.

20. Bernardo J.F., Murakami S., Branch R.A., Sabra R. Potassium depletion potentiates amphotericin B- induced toxicity to renal tubules. Nephron 1995;70:235-41. 